\title{
Some Fixed Point Theorems in Partial Fuzzy Metric Spaces
}

\author{
Başak ALDEMIR $^{1}$, Elif GÜNER ${ }^{1 *}$, Ebru AYDOĞDU ${ }^{1}$, Halis AYGÜN ${ }^{1}$
}

\begin{abstract}
The aim of this study is basically to give some fundamental fixed point theorems in partial fuzzy metric spaces. Firstly, we investigate the relationships of partial metric space and fuzzy metric space with partial fuzzy metric spaces. Then we define some contractive/contraction mappings similar to the Banach contraction mappings in the classical sense. Also, we show that these mappings have a unique fixed point under some conditions. Finally, we give some examples to illustrate the validity of the obtained results and the necessity of added conditions.
\end{abstract}

Keywords: Partial metric, partial fuzzy metric, completeness, fixed point theorem.

\footnotetext{
${ }^{1}$ Başak ALDEMİR (Orcid ID: 0000-0002-9073-9364), Elif GÜNER (Orcid ID: 0000-0002-6969-400X), Ebru AYDOĞDU (Orcid ID: 0000-0002-2777-8651), Halis AYGÜN (Orcid ID: 0000-0003-3263-3884), Kocaeli Üniversitesi, Fen Edebiyat Fakültesi, Matematik Bölümü, Kocaeli, Türkiye

*Sorumlu Yazar/Corresponding Author: Elif GÜNER, e-mail: elif.guner@kocaeli.edu.tr
}

Geliş tarihi / Received: 10-04-2020 Kabul tarihi / Accepted: 30-07-2020 


\section{INTRODUCTION}

In 1965, Zadeh initiated the theory of fuzzy set to handle the uncertainty using the membership degree. This theory has found many applications in a lot of fields since there are many real-life problems where the nature of a given system possesses fuzziness. The concept of fuzzy metric space was defined in two different ways by Kramosil and Michalek (1975) and Kaleva and Seikkala (1984). Later, George and Veeramani (1994) redefined the notion of fuzzy metric space in a slightly different way from Kramosil and Michalek to construct a Hausdorff $\left(T_{2}\right)$ topology from a given fuzzy metric space. Grabiec (1988) gave the Banach contraction theorem in the fuzzy metric setting in the means of Kramosil and Michalek (1975). Afterward, many authors (Piera, 2001, Vasuki 2003, Mihet 2004/2008, RodriguezLopez and Romaguera, 2004, Gregori et al., 2010) proved some fixed point theorems on fuzzy metric space in several senses and became interested in topological properties of fuzzy metric spaces.

In literature, there are many types of generalized metric spaces were introduced by modifying the metric axioms (Gähler 1963, Czerwik 1993, Mustafa and Sims 2006). At the same time, a lot of research has been done in these structures related to fixed point theory (Czerwik 1993, Lahiri et al. 2011, Şahin et al. 2015, Başarır and Şahin 2017). One of the generalizations of metric spaces is the notion of partial metric space which was given by Matthews (1994) as an extension of metric space where the self-distance of any point is not necessarily equal to zero. This concept is motivated with the applications to computer science. Bukatin et al. (2009) showed how the mathematics of nonzero self-distance for metric space has been established. They also considered some possible uses of partial metric spaces. Then, Valero (2005), Altun et al. (2010), Haghi et al. (2013) obtained some extensions of the result of Matthews related to Banach fixed point theorem. In the last years, Yue and Gu (2014), Sedghi et al. (2015) and Gregori et al. (2019) studied fuzzy partial metric spaces as a generalized of both partial metric space and fuzzy metric space. Yue and Gu (2014) defined the notion of fuzzy partial metric space by considering the continuous minimum t-norm. Then, Yue and Gu (2014) gave the probabilistic partial metric space is an extension of both partial metrics and fuzzy ones by the sense of $\Delta_{+}$-valued sets. They also generalized some fixed points theorems which is given by Grabiec sense and Gregori and Sapena sense to probabilistic partial metric spaces. After Sedghi et al. (2015) obtained the partial fuzzy metric space by generalizing the structure of non-Archimedean (strong) fuzzy metric and they obtained some fixed point results in this spaces. Gregori et al. (2019) approached the concept of fuzzy partial metric space by merging fuzzy metric space and partial metric space with residue operator which is pertinent to continuous t-norm. They also defined the notion of open ball and induced a $T_{0}$-topology from fuzzy partial metric.

In this work, we investigate the relationships of partial metric space and fuzzy metric space with partial fuzzy metric spaces in the sense of Sedghi et al. (2015). Then, we show that each fuzzy partial metric induces a fuzzy metric under some conditions. After, we define some contractive/contraction mappings similar to the Banach contraction mappings well-known in the fuzzy sense. As the main purpose, we show that these mappings have a unique fixed point under which conditions. Moreover, we show that some of the fixed point generalizations in partial fuzzy metric space can be induced by the pertinent result in fuzzy metric space. Finally, we give some examples to illustrate the validity of the presented results. 


\section{MATERIALS AND METHODS}

We recall some basic definitions such as fuzzy metric, partial metric, partial fuzzy metric in the sense of Sedghi et al. (2015) and some notions related in these concepts which will be useful for the main results. Also, we observe some basic properties of partial fuzzy metric spaces.

Definition 1. A partial metric space (shortly, PMS) on $U$ is a pair $(U, p)$ such that $U$ is a non-empty set and $p: U \times U \rightarrow \mathbb{R}^{+}$is a mapping providing the listed conditions for all $\mathfrak{x}, \mathfrak{y}, \mathfrak{z} \in U$ :

(PM1) $p(\mathfrak{x}, \mathfrak{x}) \leq p(\mathfrak{x}, \mathfrak{y})$,

(PM2) $p(\mathfrak{x}, \mathfrak{x})=p(\mathfrak{x}, \mathfrak{y})=p(\mathfrak{y}, \mathfrak{y})$ if and only if $\mathfrak{x}=\mathfrak{y}$,

$(\mathrm{PM} 3) p(\mathfrak{x}, \mathfrak{y})=p(\mathfrak{y}, \mathfrak{x})$,

$(\mathrm{PM} 4) p(\mathfrak{x}, \mathfrak{\jmath}) \leq p(\mathfrak{x}, \mathfrak{y})+p(\mathfrak{y}, \mathfrak{\jmath})-p(\mathfrak{y}, \mathfrak{y})$ (Matthews, 1994).

Note that the self-distance of any point is not necessarily equal to zero in partial metric space. If $p(\mathfrak{x}, \mathfrak{x})=0$ for all $\mathfrak{x} \in U$, then the partial metric $p$ is an ordinary metric on $U$. So a partial metric is a generalization of ordinary metric (Matthews, 1994).

Example 1. Let $p: \mathbb{R}^{+} \times \mathbb{R}^{+} \rightarrow \mathbb{R}^{+}$be a mapping with $p(\mathfrak{x}, \mathfrak{y})=\max \{\mathfrak{x}, \mathfrak{y}\}$ for all $\mathfrak{x}, \mathfrak{y} \in \mathbb{R}^{+}$. Then $\left(\mathbb{R}^{+}, p\right)$ is a PMS such that the self-distance of any point $x \in \mathbb{R}^{+}$is equal to itself $\left(\mathrm{O}^{\prime} \mathrm{Neill}, 1995\right)$.

Definition 2. Let $(U, p)$ be a PMS and $\left(x_{n}\right)$ be a sequence in $U$.

(i) $\left(x_{n}\right)$ is said to converge to $\mathfrak{x} \in \mathrm{U}$ if $\lim _{n \rightarrow \infty} p\left(x_{n}, \mathfrak{x}\right)=p(\mathfrak{x}, \mathfrak{x})$.

(ii) $\left(x_{n}\right)$ is said to be a Cauchy sequence if $\lim _{n, m \rightarrow \infty} p\left(x_{n}, x_{m}\right)$ exists.

(iii) $(U, p)$ is said to be complete if there is a point $\mathfrak{x} \in U$ such that $\lim _{n, m \rightarrow \infty} p\left(\mathfrak{x}_{n}, \mathfrak{x}_{m}\right)=\lim _{n \rightarrow \infty} p\left(\mathfrak{x}_{n}, \mathfrak{x}\right)=$ $p(\mathfrak{x}, \mathfrak{x})$ (Matthews, 1994).

Definition 3. Let $(U, p)$ be a complete PMS and $f$ be a self-mapping on $U$. The mapping $f$ is said to be a partial contractive mapping on $U$ if there exists a $k \in(0,1)$ such that

$$
p(f(\mathfrak{x}), f(\mathfrak{y})) \leq k p(\mathfrak{x}, \mathfrak{y})
$$

for all $\mathfrak{x}, \mathfrak{y} \in U$ (Matthews, 1994).

Theorem 1. Let $(U, p)$ be a complete PMS and $f$ be a partial contractive mapping on $U$. Then there exists a unique point $\mathfrak{x} \in U$ such that $f(\mathfrak{X})=\mathfrak{x}$. Also, $p(\mathfrak{x}, \mathfrak{X})=0$ (Matthews, 1994).

Definition 4. A binary operation $\circledast$ on $[0,1]$ is called a continuous t-norm if the listed conditions are provided for all $\mathfrak{x}, \mathfrak{y}, \mathfrak{z}, \mathfrak{w}, \in[0,1]$ :

(i) $\quad \mathfrak{x} \circledast \mathfrak{y}=\mathfrak{y} \circledast \mathfrak{x}$ and $\mathfrak{x} \circledast(\mathfrak{y} \circledast \mathfrak{z})=(\mathfrak{x} \circledast \mathfrak{y}) \circledast \mathfrak{z}$

(ii) $\circledast$ is continuous on $[0,1] \times[0,1]$,

(iii) $\mathfrak{x} \circledast 1=\mathfrak{x}$,

(iv) If $\mathfrak{x} \leq \mathfrak{y}$ and $\mathfrak{z} \leq \mathfrak{w}$, then $\mathfrak{x} \circledast \mathfrak{z} \leq \mathfrak{y} \circledast \mathfrak{w}$ (George and Veeramani, 1994).

Definition 5. Let $U$ be a nonempty set, $\circledast$ be a continuous t-norm and $\mathcal{M}: U \times U \times(0, \infty) \rightarrow[0,1]$ be a mapping. If the listed conditions are satisfied for all $\mathfrak{x}, \mathfrak{y}, \mathfrak{\mathfrak { Z }} \in U$ and $\mathfrak{t}, \mathfrak{s}>0$, then the triplet $(U, \mathcal{M}, \circledast)$ is said to be a fuzzy metric space (shortly, FMS): 
(FM1) $\mathcal{M}(\mathfrak{x}, \mathfrak{y}, \mathfrak{t})>0$,

(FM2) $\mathcal{M}(\mathfrak{x}, \mathfrak{y}, \mathfrak{t})=1$ if and only if $\mathfrak{x}=\mathfrak{y}$,

(FM3) $\mathcal{M}(\mathfrak{x}, \mathfrak{y}, \mathfrak{t})=\mathcal{M}(\mathfrak{y}, \mathfrak{x}, \mathfrak{t})$,

(FM4) $\mathcal{M}(\mathfrak{x}, \mathfrak{\jmath}, \mathfrak{t}+\mathfrak{s}) \geq \mathcal{M}(\mathfrak{x}, \mathfrak{y}, \mathfrak{t}) \circledast \mathcal{M}(\mathfrak{y}, \mathfrak{\jmath}, \mathfrak{s})$,

(FM5) $\mathcal{M}(\mathfrak{x}, \mathfrak{y}, \cdot)$ is continuous on $(0, \infty)$ (George and Veeramani, 1994).

If a FMS $(U, \mathcal{M}, \circledast)$ provide the following condition for all $\mathfrak{x}, \mathfrak{y}, \mathfrak{z} \in U$ and $\mathfrak{s}, \mathrm{t}>0$, then $(U, \mathcal{M}, \circledast)$ is said to be a non-Archimedean (strong) FMS

$$
\mathcal{M}(\mathfrak{x}, \mathfrak{y}, \max \{\mathrm{t}, \mathfrak{s}\}) \geq \mathcal{M}(\mathfrak{x}, \mathfrak{z}, \mathrm{t}) \circledast \mathcal{M}(\mathfrak{z}, \mathfrak{y}, \mathfrak{s})
$$

(Gregori et al., 2010)

Proposition 1. If $(U, \mathcal{M}, \circledast)$ is a FMS, then $\mathcal{M}(\mathfrak{x}, \mathfrak{y}) \cdot):(0, \infty) \rightarrow[0,1]$ is non-decreasing for all $\mathfrak{x}, \mathfrak{y} \in U$ (George and Veeramani, 1994).

Definition 6. Let $(U, \mathcal{M}, \circledast)$ be a FMS and $\left(x_{n}\right)$ be a sequence in $U$.

(i) $\quad\left(x_{n}\right)$ is said to converge to $x \in U$ if $\mathcal{M}\left(x_{n}, \mathfrak{x}, \mathrm{t}\right) \rightarrow 1$ as $n \rightarrow \infty$ for all $\mathrm{t}>0$.

(ii) $\quad\left(\mathfrak{x}_{n}\right)$ is said to be a Cauchy sequence if $\mathcal{M}\left(\mathfrak{x}_{n}, \mathfrak{x}_{m}, \mathrm{t}\right) \rightarrow 1$ as $n, m \rightarrow \infty$ for all $\mathrm{t}>0$.

(iii) If each Cauchy sequence $\left(x_{n}\right)$ converges to a point $\mathfrak{x} \in U$, then $(U, \mathcal{M}, \circledast)$ is called complete (George and Veeramani, 1994).

Theorem 2. Let $(U, \mathcal{M}, \circledast)$ be a FMS. If $f$ is a self-mapping on $U$ satisfying $\mathcal{F}(f(\mathfrak{x}), f(\mathfrak{y}), \mathfrak{t})>\mathcal{F}(\mathfrak{x}, \mathfrak{y}, \mathfrak{t})$ for all $\mathfrak{x}, \mathfrak{y} \in U(\mathfrak{x} \neq \mathfrak{y})$ and $\mathfrak{t}>0$ and there is a point $\mathfrak{x}_{0} \in U$ whose sequence of iterates $\left(f^{n}\left(\mathfrak{x}_{0}\right)\right)$ contains a convergent subsequence $\left(f^{n_{i}}\left(\mathfrak{x}_{0}\right)\right)$, then $f$ has a unique fixed point in $U$ (Grabiec, 1988).

Partial fuzzy metric space was defined by Sedghi et al. (2015) as a generalization of partial metric and fuzzy metric spaces:

Definition 7. Let $U$ be a nonempty set, $\circledast$ be a continuous t-norm and $\mathcal{F}: U \times U \times(0, \infty) \rightarrow[0,1]$ be a mapping. If the listed conditions are satisfied for all $\mathfrak{x}, \mathfrak{y}, \mathfrak{z} \in U$ and $t, \mathfrak{s}>0$, then the triplet $(U, \mathcal{F}, \circledast)$ is said to be a partial fuzzy metric space (shortly, PFMS) :

(PFM1) $\quad \mathfrak{x}=\mathfrak{y}$ if and only if $\mathcal{F}(\mathfrak{x}, \mathfrak{y}, \mathfrak{t})=\mathcal{F}(\mathfrak{x}, \mathfrak{x}, \mathfrak{t})=\mathcal{F}(\mathfrak{y}, \mathfrak{y}, \mathfrak{t})$,

(PFM2) $\quad \mathcal{F}(\mathfrak{x}, \mathfrak{x}, \mathrm{t}) \geq \mathcal{F}(\mathfrak{x}, \mathfrak{y}, \mathrm{t})>0$,

(PFM3) $\quad \mathcal{F}(\mathfrak{x}, \mathfrak{y}, \mathfrak{t})=\mathcal{F}(\mathfrak{y}, \mathfrak{x}, \mathrm{t})$,

(PFM4) $\quad \mathcal{F}(\mathfrak{x}, \mathfrak{\jmath}, \mathfrak{t}) \circledast F(\mathfrak{z}, \mathfrak{y}, \mathfrak{s}) \leq \mathcal{F}(x, \mathfrak{y}, \max \{\mathrm{t}, \mathfrak{s}\}) \circledast \mathcal{F}(\mathfrak{\jmath}, \mathfrak{\jmath}, \max \{\mathrm{t}, \mathfrak{s}\})$,

(PFM5) $\quad \mathcal{F}(\mathfrak{x}, \mathfrak{y}, \cdot)$ is continuous on $(0, \infty)$ (Sedghi et al., 2015).

Remark 1. Let $(U, \mathcal{F}, \circledast)$ be a PFMS.

(1) If $\mathcal{F}(\mathfrak{x}, \mathfrak{y}, \mathfrak{t})=1$, then $\mathfrak{x}=\mathfrak{y}$ from the conditions (PFM1) and (PFM2). But the converse of this implication need not be necessarily true. i.e., $\mathcal{F}(\mathfrak{x}, \mathfrak{y}, \mathfrak{t})$ may not be equal to 1 whenever $\mathfrak{x}=\mathfrak{y}$.

(2) It is clear that

$$
\mathcal{F}(\mathfrak{x}, \mathfrak{z}, \mathfrak{t}) \circledast \mathcal{F}(\mathfrak{z}, \mathfrak{y}, \mathfrak{t}) \leq \mathcal{F}(\mathfrak{x}, \mathfrak{y}, \mathfrak{t}) \circledast \mathcal{F}(\mathfrak{z}, \mathfrak{z}, \mathfrak{t})
$$

for all $\mathfrak{x}, \mathfrak{y}, \mathfrak{z} \in U$ and $\mathfrak{t}>0$ from the conditions (PFM4) (Sedghi et al., 2015).

Note that each non-Archimedean FMS is a PFMS, but the converse implication may not be true. 
Example 2. Let $(U, p)$ be a PMS and $\mathfrak{a} \circledast \mathfrak{b}=\mathfrak{a} \mathfrak{b}$ for all $\mathfrak{a}, \mathfrak{b} \in[0,1]$. Consider the mapping $\mathcal{F}_{p}: U \times U \times(0, \infty) \rightarrow[0,1]$ defined by

$$
\mathcal{F}_{p}(\mathfrak{x}, \mathfrak{y}, \mathfrak{t})=\frac{\mathrm{t}}{\mathrm{t}+p(\mathfrak{x}, \mathfrak{y})} .
$$

Then $\left(U, \mathcal{F}_{p}, \circledast\right)$ is a PFMS which is called the standard PFMS. Note that $\left(U, \mathcal{F}_{p}, \circledast\right)$ is not a FMS (Sedghi et al., 2015).

There are some difference between PFMS and FMS. One of them, in a FMS $(U, \mathcal{M}, \circledast)$, $\mathcal{M}(\mathfrak{x}, \mathfrak{y}, \cdot):(0, \infty) \rightarrow[0,1]$ is non-decreasing function for all $\mathfrak{x}, \mathfrak{y} \in U$, but in a PFMS $(U, \mathcal{F}, \circledast)$, $\mathcal{F}(\mathfrak{x}, \mathfrak{y}, \cdot):(0, \infty) \rightarrow[0,1]$ may not be non-decreasing function for all $\mathfrak{x}, \mathfrak{y} \in U$.

In the following example, we show that there are partial fuzzy metric spaces $(U, \mathcal{F}, \circledast)$ in which $\mathcal{F}(\mathfrak{x}, \mathfrak{y}, \cdot):(0, \infty) \rightarrow[0,1]$ may not be non-decreasing function for all $\mathfrak{x}, \mathfrak{y} \in U$.

Example 3. Let $U=\mathbb{R}$ and $\mathfrak{a} \circledast \mathfrak{b}=\min \{\mathfrak{a}, \mathfrak{b}\}$ for all $\mathfrak{a}, \mathfrak{b} \in[0,1]$. Consider the mapping $\mathcal{F}: U \times U \times$ $(0, \infty) \rightarrow[0,1]$ defined by

$$
\mathcal{F}(\mathfrak{x}, \mathfrak{y}, \mathfrak{t})= \begin{cases}e^{-t}, & \mathfrak{x}=\mathfrak{y} \\ \frac{1}{3} e^{-t}, & \mathfrak{x} \neq \mathfrak{y} .\end{cases}
$$

It is not complicated to see that $(U, \mathcal{F}, \circledast)$ is a PFMS. Concretely, (PFM1)-(PFM3) are obviously hold by the definition of $\mathcal{F}$. Now, we show that (PFM4) is satisfied. Let $x, \mathfrak{y}, \mathfrak{z} \in U$ and $\mathfrak{x} \neq \mathfrak{z}$ (for the contrary since $e^{-\mathrm{t}} \geq \frac{1}{4} e^{-\mathrm{t}}$, the inequality is fullfilled). Assume $\mathfrak{s}<\mathrm{t}$. For $\mathfrak{y} \neq \mathfrak{x}$ and $\mathfrak{y} \neq \mathfrak{z}$, we obtain

$$
\begin{aligned}
\mathcal{F}(\mathfrak{x}, \mathfrak{z}, \mathfrak{t}) \circledast F(\mathfrak{z}, \mathfrak{y}, \mathfrak{s}) & =\min \left\{\frac{1}{3} e^{-\mathrm{t}}, \frac{1}{3} e^{-s}\right\}=\frac{1}{3} e^{-\mathrm{t}}=\min \left\{\frac{1}{3} e^{-\mathrm{t}}, e^{-t}\right\} \\
& =\mathcal{F}(\mathfrak{x}, \mathfrak{y}, \max \{\mathrm{t}, \mathfrak{s}\}) \circledast \mathcal{F}(\mathfrak{z}, \mathfrak{\jmath}, \max \{\mathrm{t}, \mathfrak{s}\}) .
\end{aligned}
$$

Let $\mathfrak{y}=\mathfrak{x}$ and $\mathfrak{y} \neq \mathfrak{z}$, then we get

$$
\begin{aligned}
\mathcal{F}(\mathfrak{x}, \mathfrak{\jmath}, \mathfrak{t}) \circledast F(\mathfrak{z}, \mathfrak{y}, \mathfrak{s}) & =\min \left\{\frac{1}{3} e^{-\mathrm{t}}, \frac{1}{3} e^{-s}\right\}=\frac{1}{3} e^{-\mathrm{t}} \leq \min \left\{e^{-\mathrm{t}}, e^{-t}\right\} \\
= & \mathcal{F}(\mathfrak{x}, \mathfrak{y}, \max \{\mathrm{t}, \mathfrak{s}\}) \circledast \mathcal{F}(\mathfrak{3}, \mathfrak{\jmath}, \max \{\mathrm{t}, \mathfrak{s}\}) .
\end{aligned}
$$

Let $\mathfrak{y}=\mathfrak{z}$ and $\mathfrak{y} \neq \mathfrak{x}$, then we get

$$
\begin{aligned}
\mathcal{F}(\mathfrak{x}, \mathfrak{\jmath}, \mathfrak{t}) \circledast F(\mathfrak{z}, \mathfrak{y}, \mathfrak{s}) & =\min \left\{\frac{1}{3} e^{-\mathrm{t}}, e^{-s}\right\}=\frac{1}{3} e^{-\mathrm{t}}=\min \left\{\frac{1}{3} e^{-\mathrm{t}}, e^{-t}\right\} \\
= & \mathcal{F}(\mathfrak{x}, \mathfrak{y}, \max \{\mathrm{t}, \mathfrak{s}\}) \circledast \mathcal{F}(\mathfrak{z}, \mathfrak{\jmath}, \max \{\mathrm{t}, \mathfrak{s}\}) .
\end{aligned}
$$

Finally, $\mathcal{F}(\mathfrak{x}, \mathfrak{y}, \cdot)$ is continuous on $(0, \infty)$. Hence $(U, \mathcal{F}, \circledast)$ is a PFMS. Also, it is obvious that $\mathcal{F}(x, \mathfrak{y}, \cdot)$ : $(0, \infty) \rightarrow[0,1]$ is a decreasing function.

Proposition 2. Let $(U, \mathcal{F}, \circledast)$ be a PFMS. If $\mathfrak{b} \geq \mathfrak{c}$ whenever $\mathfrak{a} \circledast \mathfrak{b} \geq \mathfrak{a} \circledast \mathfrak{c}$ for all $\mathfrak{a}, \mathfrak{b}, \mathfrak{c} \in[0,1]$, then $\mathcal{F}(\mathfrak{x}, \mathfrak{y}, \cdot):(0, \infty) \rightarrow[0,1]$ is non-decreasing function for all $\mathfrak{x}, \mathfrak{y} \in U$. (Sedghi et al., 2015).

Definition 8: Let $(U, \mathcal{F}, \circledast)$ be a PFMS and $\left(x_{n}\right)$ be a sequence in $U$.

(i) $\left(x_{n}\right)$ is said to converge to a point $\mathfrak{x} \in \mathrm{U}$ if $\lim _{n \rightarrow \infty} \mathcal{F}\left(\mathfrak{x}_{n}, \mathfrak{x}, \mathrm{t}\right)=\mathcal{F}(\mathfrak{x}, \mathfrak{x}, \mathrm{t})$ for all $\mathrm{t}>0$ (Sedghi et al., 2015). 
(ii) $\left(\mathfrak{x}_{n}\right)$ is said to be a Cauchy sequence if $\lim _{n, m \rightarrow \infty} \mathcal{F}\left(\mathfrak{x}_{n}, \mathfrak{x}_{m}, \mathrm{t}\right)$ exists for all $\mathrm{t}>0$. If $\lim _{n, m \rightarrow \infty} \mathcal{F}\left(\mathfrak{x}_{n}, \mathfrak{x}_{m}, \mathfrak{t}\right)=1$ then $\left(\mathfrak{x}_{n}\right)$ is called a 1-Cauchy sequence.

(iii) If each Cauchy sequence (resp. 1-Cauchy) $\left(x_{n}\right)$ converges to a point $\mathfrak{X} \in U$ such that $\lim _{n, m \rightarrow \infty} \mathcal{F}\left(\mathfrak{x}_{n}, \mathfrak{x}_{m}, \mathfrak{t}\right)=\mathcal{F}(\mathfrak{x}, \mathfrak{x}, \mathrm{t})$, then $(U, \mathcal{F}, \circledast)$ is said to be complete (resp. 1-complete).

Clearly, every 1-Cauchy sequence $\left(x_{n}\right)$ in $(U, \mathcal{F}, \circledast)$ is also a Cauchy sequence and every complete PFMS is a 1-complete space.

Proposition 3. Let $(U, \mathcal{F}, \circledast)$ be a PFMS and $\left(x_{n}\right)$ be a sequence in $U$ such that $\lim _{n \rightarrow \infty} \mathcal{F}\left(\mathfrak{x}_{n}, \mathfrak{x}_{n}, \mathfrak{t}\right)=\lim _{n \rightarrow \infty} \mathcal{F}\left(\mathfrak{x}_{n}, \mathfrak{x}, \mathfrak{t}\right)=\mathcal{F}(\mathfrak{x}, \mathfrak{x}, \mathfrak{t})$. If $\mathfrak{b} \geq \mathfrak{c}$ whenever $\mathfrak{a} \circledast \mathfrak{b} \geq \mathfrak{a} \circledast \mathfrak{c}$ for all $\mathfrak{a}, \mathfrak{b}, \mathfrak{c} \in[0,1]$, then $\lim _{n \rightarrow \infty} \mathcal{F}\left(\mathfrak{x}_{n}, \mathfrak{y}, \mathfrak{t}\right)=F(\mathfrak{x}, \mathfrak{y}, \mathfrak{t})$ for all $\mathfrak{y} \in U$ and $\mathfrak{t}>0$ (Sedghi et al., 2015).

\section{RESULTS AND DISCUSSION}

In this section, we define partial fuzzy (Edelstein) contractive/contraction and partial fuzzy $\psi$-contractive mappings in PFMS which are analogous in FMS given by Grabiec (1988), Gregori and Sapena (2002) and Mihet (2008), respectively. Then we present some fixed point results for given selfmappings in PFMS and present several examples to show the validity of obtained results.

Proposition 4. Let $(U, p)$ be a PMS and $\left(U, \mathcal{F}_{p}, \circledast\right)$ be a standard PFMS induced by this $p$.

(1) $\left(\mathfrak{x}_{n}\right)$ converges to $\mathfrak{x} \in U$ in $\left(U, \mathcal{F}_{p}, \circledast\right)$ if and only if $\left(\mathfrak{x}_{n}\right)$ converges to $\mathfrak{x} \in U$ in $(U, p)$.

(2) $\left(\mathfrak{x}_{n}\right)$ is a Cauchy sequence in $\left(U, \mathcal{F}_{p}, \circledast\right)$ if and only if $\left(\mathfrak{x}_{n}\right)$ is a Cauchy sequence in $(U, p)$.

(3) $\left(U, \mathcal{F}_{p}, \circledast\right)$ is complete if and only if $(U, p)$ is complete. Furthermore, $\lim _{n \rightarrow \infty} p\left(x_{n}, \mathfrak{X}\right)=p(\mathfrak{x}, \mathfrak{x})=$ $\lim _{n, m \rightarrow \infty} p\left(\mathfrak{x}_{n}, \mathfrak{x}_{m}\right)$ if and only if $\lim _{n \rightarrow \infty} \mathcal{F}_{p}\left(\mathfrak{x}_{n}, \mathfrak{x}, \mathfrak{t}\right)=\mathcal{F}_{p}(\mathfrak{x}, \mathfrak{x}, \mathfrak{t})=\lim _{n, m \rightarrow \infty} \mathcal{F}_{p}\left(\mathfrak{x}_{n}, \mathfrak{x}_{m}, \mathfrak{t}\right)$.

Proof. (1) Let $\left(x_{n}\right)$ converges to $\mathfrak{x} \in U$ in $\left(U, \mathcal{F}_{p}, \circledast\right)$. Then we have $\lim _{n \rightarrow \infty} \mathcal{F}_{p}\left(x_{n}, \mathfrak{x}, \mathfrak{t}\right)=\mathcal{F}_{p}(\mathfrak{x}, \mathfrak{x}, \mathrm{t})$. It means that $\lim _{n \rightarrow \infty} \frac{t}{\mathfrak{t}+p(\mathfrak{x}, \mathfrak{y})}=\frac{\mathrm{t}}{\mathrm{t}+p(\mathfrak{x}, \mathfrak{x})}$. Hence, we get $\lim _{n \rightarrow \infty} p\left(\mathfrak{x}_{n}, \mathfrak{x}\right)=p(\mathfrak{x}, \mathfrak{x})$. Therefore, $\left(\mathfrak{x}_{n}\right)$ converges to $\mathfrak{x} \in$ $U$ in $(U, p)$.

(2) Let $\left(\mathfrak{x}_{n}\right)$ be a Cauchy sequence in $\left(U, \mathcal{F}_{p}, \circledast\right)$. Then $\lim _{n, m \rightarrow \infty} \mathcal{F}_{p}\left(\mathfrak{x}_{n}, \mathfrak{x}_{m}, \mathrm{t}\right)$ exists. Thus

$$
\lim _{n, m \rightarrow \infty} \mathcal{F}_{p}\left(\mathfrak{x}_{n}, \mathfrak{x}_{m}, \mathrm{t}\right)=\lim _{n, m \rightarrow \infty} \frac{\mathrm{t}}{\mathrm{t}+p\left(\mathfrak{x}_{n}, \mathfrak{x}_{m}\right)}=\frac{\mathrm{t}}{\mathrm{t}+\lim _{n, m \rightarrow \infty} p\left(\mathfrak{x}_{n}, \mathfrak{x}_{m}\right)}
$$

This means $\lim _{n, m \rightarrow \infty} p\left(\mathfrak{x}_{n}, \mathfrak{x}_{m}\right)$ exists. Hence $\left(\mathfrak{x}_{n}\right)$ is a Cauchy sequence in $(U, p)$. The converse statement is similar.

(3) Let $\left(U, \mathcal{F}_{p}, \circledast\right)$ be a complete PFMS and $\left(\mathfrak{x}_{n}\right)$ be a Cauchy sequence in $(U, p)$. By $(2),\left(x_{n}\right)$ is also a Cauchy sequence in $\left(U, \mathcal{F}_{p}, \circledast\right)$. Since $\left(U, \mathcal{F}_{p}, \circledast\right)$ is complete, we have $\left(\mathfrak{x}_{n}\right)$ is convergent in $\left(U, \mathcal{F}_{p}, \circledast\right)$. By $(1),\left(x_{n}\right)$ is also convergent in $(U, p)$. Thus $(U, p)$ is a complete PMS. The other side is obtained similarly. The equality of limits is obvious by the definition of $\mathcal{F}_{p}$.

Theorem 3: Let $(U, \mathcal{F}, \circledast)$ be a PFMS such that $\mathcal{F}(x, \mathfrak{y}, \cdot):(0, \infty) \rightarrow[0,1]$ is non-decreasing function for all $\mathfrak{x}, \mathfrak{y} \in U$. Then the mapping $\mathcal{M}_{\mathcal{F}}: U \times U \times(0, \infty) \rightarrow[0,1]$ defined by $\mathcal{M}_{\mathcal{F}}(\mathfrak{x}, \mathfrak{y}, \mathfrak{t})=1$ whenever 
$\mathfrak{X}=\mathfrak{y}$ and $\mathcal{M}_{\mathcal{F}}(\mathfrak{x}, \mathfrak{y}, \mathfrak{t})=\mathcal{F}(\mathfrak{x}, \mathfrak{y}, \mathfrak{t})$ whenever $\mathfrak{x} \neq \mathfrak{y}$, is a fuzzy metric on $U$. Moreover, $\left(U, \mathcal{M}_{\mathcal{F}}, \circledast\right)$ is complete if and only if $(U, \mathcal{F}, \circledast)$ is 1-complete.

Proof: It is clear that (FM1), (FM2), (FM3) and (FM5) are satisfied. Now, we only show that (FM4) is satisfied. Let $\mathfrak{x}, \mathfrak{y}, \mathfrak{z} \in U$. It is obvious that (FM4) is satisfied whenever $\mathfrak{x}, \mathfrak{y}, \mathfrak{z}$ are equal.

Let $\mathfrak{x} \neq \mathfrak{y} \neq \mathfrak{z}$, then

$$
\begin{aligned}
\mathcal{M}_{\mathcal{F}}(\mathfrak{x}, \mathfrak{y}, \mathfrak{t}) \circledast \mathcal{M}_{\mathcal{F}}(\mathfrak{y}, \mathfrak{\jmath}, \mathfrak{s}) & =\mathcal{F}(\mathfrak{x}, \mathfrak{y}, \mathfrak{t}) \circledast \mathcal{F}(\mathfrak{y}, \mathfrak{z}, \mathfrak{s}) \leq \mathcal{F}(\mathfrak{x}, \mathfrak{z}, \max \{\mathrm{t}, \mathfrak{s}\}) \circledast \mathcal{F}(\mathfrak{y}, \mathfrak{y}, \max \{\mathrm{t}, \mathfrak{s}\}) \\
& \leq \mathcal{F}(\mathfrak{x}, \mathfrak{z}, \max \{\mathrm{t}, \mathfrak{s}\}) \leq \mathcal{F}(\mathfrak{x}, \mathfrak{z}, \mathrm{t}+\mathfrak{s})=\mathcal{M}_{\mathcal{F}}(\mathfrak{x}, \mathfrak{3}, \mathrm{t}+\mathfrak{s}) .
\end{aligned}
$$

Let $\mathfrak{x} \neq \mathfrak{y}$ and $\mathfrak{x}=\mathfrak{z}$, then

$$
\begin{aligned}
\mathcal{M}_{\mathcal{F}}(\mathfrak{x}, \mathfrak{y}, \mathfrak{t}) \circledast \mathcal{M}_{\mathcal{F}}(\mathfrak{y}, \mathfrak{z}, \mathfrak{s}) & =\mathcal{F}(\mathfrak{x}, \mathfrak{y}, \mathfrak{t}) \circledast \mathcal{F}(\mathfrak{y}, \mathfrak{z}, \mathfrak{s}) \leq \mathcal{F}(\mathfrak{x}, \mathfrak{z}, \max \{\mathrm{t}, \mathfrak{s}\}) \circledast \mathcal{F}(\mathfrak{y}, \mathfrak{y}, \max \{\mathrm{t}, \mathfrak{s}\}) \\
& =\mathcal{F}(\mathfrak{x}, \mathfrak{x}, \max \{\mathrm{t}, \mathfrak{s}\}) \circledast \mathcal{F}(\mathfrak{y}, \mathfrak{y}, \max \{\mathrm{t}, \mathfrak{s}\}) \leq 1=\mathcal{M}_{\mathcal{F}}(\mathfrak{x}, \mathfrak{z}, \mathrm{t}+\mathfrak{s}) .
\end{aligned}
$$

Let $\mathfrak{x} \neq \mathfrak{y}$ and $\mathfrak{y}=\mathfrak{z}$, then

$$
\mathcal{M}_{\mathcal{F}}(\mathfrak{x}, \mathfrak{y}, \mathfrak{t}) \circledast \mathcal{M}_{\mathcal{F}}(\mathfrak{y}, \mathfrak{z}, \mathfrak{s})=\mathcal{F}(\mathfrak{x}, \mathfrak{y}, \mathfrak{t}) \circledast 1=\mathcal{F}(\mathfrak{x}, \mathfrak{y}, \mathfrak{t}) \leq \mathcal{F}(\mathfrak{x}, \mathfrak{z}, \mathfrak{t}+\mathfrak{s})=\mathcal{M}_{\mathcal{F}}(\mathfrak{x}, \mathfrak{\jmath}, \mathfrak{t}+\mathfrak{s}) .
$$

Therefore, we have that $\left(U, \mathcal{M}_{\mathcal{F}}, \circledast\right)$ is a fuzzy metric space.

Let $\left(U, \mathcal{M}_{\mathcal{F}}, \circledast\right)$ be a complete FMS and $\left(x_{n}\right)$ be a 1 -Cauchy sequence in $(U, \mathcal{F}, \circledast)$. Thus $\lim _{n, m \rightarrow \infty} \mathcal{F}\left(\mathfrak{x}_{n}, \mathfrak{x}_{m}, \mathfrak{t}\right)=1$. Wlog, we assume that $\mathfrak{x}_{n} \neq \mathfrak{x}_{m}$ for all $n \neq m$. Then

$\lim _{n, m \rightarrow \infty} \mathcal{M}_{\mathcal{F}}\left(\mathfrak{x}_{n}, \mathfrak{x}_{m}, \mathfrak{t}\right)=1$. This means that $\left(\mathfrak{x}_{n}\right)$ is a Cauchy sequence in $\left(U, \mathcal{M}_{\mathcal{F}}, \circledast\right)$. Since $\left(U, \mathcal{M}_{\mathcal{F}}, \circledast\right)$ is complete, there is a point $\mathfrak{x} \in U$ such that $\lim _{n \rightarrow \infty} \mathcal{M}_{\mathcal{F}}\left(\mathfrak{x}_{n}, \mathfrak{x}, \mathrm{t}\right)=1$. Since $\mathcal{M}_{\mathcal{F}}(\mathfrak{x}, \mathfrak{x}, \mathrm{t})=1$, we obtain that $\lim _{n \rightarrow \infty} \mathcal{F}\left(\mathfrak{x}_{n}, \mathfrak{x}, \mathrm{t}\right)=1=\mathcal{F}(\mathfrak{x}, \mathfrak{x}, \mathrm{t})$. Hence $(U, \mathcal{F}, \circledast)$ is a 1-complete PFMS.

Conversely, assume that $(U, \mathcal{F}, \circledast)$ is a 1-complete PFMS and $\left(x_{n}\right)$ is a Cauchy sequence in $\left(U, \mathcal{M}_{\mathcal{F}}, \circledast\right)$. Then $\lim _{n, m \rightarrow \infty} \mathcal{M}_{\mathcal{F}}\left(\mathfrak{x}_{n}, \mathfrak{x}_{m}, \mathfrak{t}\right)=1$ and then $\lim _{n, m \rightarrow \infty} \mathcal{F}\left(\mathfrak{x}_{n}, \mathfrak{x}_{m}, \mathfrak{t}\right)=1$. Thus $\left(\mathfrak{x}_{n}\right)$ is a 1-Cauchy sequence in $(U, \mathcal{F}, \circledast)$. Since $(U, \mathcal{F}, \circledast)$ is a 1 -complete PFMS, there is a point $\mathfrak{x} \in U$ such that $\lim _{n \rightarrow \infty} \mathcal{F}\left(x_{n}, \mathfrak{x}, \mathfrak{t}\right)=1=\mathcal{F}(\mathfrak{x}, \mathfrak{x}, \mathfrak{t})$. Thus $\lim _{n \rightarrow \infty} \mathcal{M}_{\mathcal{F}}\left(x_{n}, \mathfrak{x}, \mathfrak{t}\right)=1$. Therefore, $\left(x_{n}\right)$ is convergent to $\mathfrak{x} \in U$ in $\left(U, \mathcal{M}_{\mathcal{F}}, \circledast\right)$. Consequently $\left(U, \mathcal{M}_{\mathcal{F}}, \circledast\right)$ is a complete FMS.

Denote by $\Psi$ the collection of all self-mappings $\psi$ on $[0,1]$ such that $\psi$ is continuous, nondecreasing and $\mathrm{t}<\psi(\mathrm{t})$ for each $t \in(0,1)$.

Definition 9. Let $(U, \mathcal{F}, \circledast)$ be a PFMS and $\psi \in \Psi$. A self-mapping $f$ on $U$ is called

$$
\text { partial fuzzy contraction if there exists a } k \in(0,1) \text { such that }
$$

$$
\mathcal{F}(f(\mathfrak{x}), f(\mathfrak{y}), k \mathrm{t}) \geq \mathcal{F}(\mathfrak{x}, \mathfrak{y}, \mathfrak{t})
$$

for all $\mathfrak{x}, \mathfrak{y} \in U$ and $\mathrm{t}>0$.

(ii) partial fuzzy Edelstein contractive if

$$
\mathcal{F}(f(\mathfrak{x}), f(\mathfrak{y}), \mathfrak{t})>\mathcal{F}(\mathfrak{x}, \mathfrak{y}, \mathfrak{t})
$$

for all $\mathfrak{x}, \mathfrak{y} \in U(\mathfrak{x} \neq \mathfrak{y})$ and $\mathfrak{t}>0$.

(iii) partial fuzzy contractive if there exists a $k \in(0,1)$ such that

$$
k\left(\frac{1}{\mathcal{F}(\mathfrak{x}, \mathfrak{y}, \mathrm{t})}-1\right) \geq \frac{1}{\mathcal{F}(f(\mathfrak{x}), f(\mathfrak{y}), \mathfrak{t})}-1
$$

for all $\mathfrak{x}, \mathfrak{y} \in U$ and $\mathrm{t}>0$.

(iv) partial fuzzy $\psi$-contractive if

$$
\mathcal{F}(f(\mathfrak{x}), f(\mathfrak{y}), \mathfrak{t}) \geq \psi(\mathcal{F}(\mathfrak{x}, \mathfrak{y}, \mathfrak{t}))
$$

for all $\mathfrak{x}, \mathfrak{y} \in U$ and $\mathrm{t}>0$. 
Proposition 5. (i) Every partial fuzzy contractive mapping is a partial fuzzy Edelstein contractive.

(ii) Every partial fuzzy contractive mapping is a partial fuzzy $\psi$-contractive.

(iii) If $(U, \mathcal{F}, \circledast)$ is a PFMS such that $\mathcal{F}(x, \mathfrak{y}, \cdot):(0, \infty) \rightarrow[0,1]$ is a non-decreasing function for all $\mathfrak{x}, \mathfrak{y} \in U$, then every partial fuzzy contraction mapping is a partial fuzzy Edelstein contractive.

Proof. It is clear from the Definition 9.

Theorem 4. Let $(U, \mathcal{F}, \circledast)$ be a complete PFMS such that $\lim _{t \rightarrow \infty} \mathcal{F}(\mathfrak{x}, \mathfrak{y}, \mathfrak{t})=1$ for all $\mathfrak{x}, \mathfrak{y} \in U$. If a selfmapping $f$ on $U$ is a partial fuzzy contraction mapping, then $f$ has a unique fixed point in $U$.

Proof. Let $\mathfrak{x}_{0} \in U$ and $\mathfrak{x}_{n}=f^{n}\left(\mathfrak{x}_{0}\right)$ for all $n \in \mathbb{N}$. Since $\lim _{t \rightarrow \infty} \mathcal{F}(\mathfrak{x}, \mathfrak{y}, \mathfrak{t})=1$ for all $\mathfrak{x}, \mathfrak{y} \in U$, then we have

$$
\begin{gathered}
\mathcal{F}\left(\mathfrak{x}_{n+1}, \mathfrak{x}_{n}, \mathfrak{t}\right)=\mathcal{F}\left(f\left(\mathfrak{x}_{n}\right), f\left(\mathfrak{x}_{n-1}\right), \mathfrak{t}\right) \geq \mathcal{F}\left(\mathfrak{x}_{n}, \mathfrak{x}_{n-1}, \frac{\mathfrak{t}}{k}\right)=\mathcal{F}\left(f\left(\mathfrak{x}_{n-1}\right), f\left(\mathfrak{x}_{n-2}\right), \frac{\mathfrak{t}}{k}\right) \\
\geq \mathcal{F}\left(\mathfrak{x}_{n-1}, \mathfrak{x}_{n-2}, \frac{\mathfrak{t}}{k^{2}}\right) \geq \cdots \geq \mathcal{F}\left(\mathfrak{x}_{1}, \mathfrak{x}_{0}, \frac{\mathfrak{t}}{k^{n}}\right) \stackrel{n \rightarrow \infty}{\longrightarrow} 1
\end{gathered}
$$

for all $\mathrm{t}>0$. Therefore, we get $\lim _{n \rightarrow \infty} \mathcal{F}\left(x_{n+1}, \mathfrak{x}_{n}, \mathrm{t}\right)=1$ for all $\mathrm{t}>0$.

Let $n, m \in \mathbb{N}$ and we may assume that $n<m$.

$$
\begin{aligned}
\mathcal{F}\left(\mathfrak{x}_{n}, \mathfrak{x}_{m}, \mathrm{t}\right) & \geq \mathcal{F}\left(\mathfrak{x}_{n}, \mathfrak{x}_{m}, \mathrm{t}\right) \circledast \mathcal{F}\left(\mathfrak{x}_{n+1}, \mathfrak{x}_{n+1}, \mathrm{t}\right) \geq \mathcal{F}\left(\mathfrak{x}_{n}, \mathfrak{x}_{n+1}, \mathrm{t}\right) \circledast \mathcal{F}\left(\mathfrak{x}_{m}, \mathfrak{x}_{n+1}, \mathrm{t}\right) \\
& \geq \mathcal{F}\left(\mathfrak{x}_{n}, \mathfrak{x}_{n+1}, \mathrm{t}\right) \circledast \mathcal{F}\left(\mathfrak{x}_{m}, \mathfrak{x}_{n+1}, \mathrm{t}\right) \circledast \mathcal{F}\left(\mathfrak{x}_{n+2}, \mathfrak{x}_{n+2}, \mathrm{t}\right) \\
& \geq \mathcal{F}\left(\mathfrak{x}_{n}, \mathfrak{x}_{n+1}, \mathrm{t}\right) \circledast \mathcal{F}\left(\mathfrak{x}_{n+1}, \mathfrak{x}_{n+2}, \mathrm{t}\right) \circledast \mathcal{F}\left(\mathfrak{x}_{n+2}, \mathfrak{x}_{m}, \mathrm{t}\right) \\
& \geq \cdots \geq \mathcal{F}\left(\mathfrak{x}_{n}, \mathfrak{x}_{n+1}, \mathrm{t}\right) \circledast \mathcal{F}\left(\mathfrak{x}_{n+1}, \mathfrak{x}_{n+2}, \mathrm{t}\right) \circledast \ldots \circledast \mathcal{F}\left(\mathfrak{x}_{m-1}, \mathfrak{x}_{m}, \mathrm{t}\right)
\end{aligned}
$$

Thus, we obtain $\lim _{n, m \rightarrow \infty} \mathcal{F}\left(\mathfrak{x}_{n}, \mathfrak{x}_{m}, \mathrm{t}\right)=1$ for all $\mathrm{t}>0$. Hence $\left(\mathfrak{x}_{n}\right)$ is a Cauchy sequence in $(U, \mathcal{F}, \circledast)$. Since $(U, \mathcal{F}, \circledast)$ is a complete PFMS, there exists a point $\mathfrak{X} \in U$ such that $\left(\mathfrak{x}_{n}\right)$ converges to $\mathfrak{x}$. Besides, $\lim _{n \rightarrow \infty} \mathcal{F}\left(\mathfrak{x}_{n}, \mathfrak{x}, \mathrm{t}\right)=\mathcal{F}(\mathfrak{x}, \mathfrak{x}, \mathrm{t})=\lim _{n, m \rightarrow \infty} \mathcal{F}\left(\mathfrak{x}_{n}, \mathfrak{x}_{m}, \mathfrak{t}\right)=1$ for all $\mathrm{t}>0$.

Then,

$$
\begin{aligned}
\mathcal{F}(f(\mathfrak{x}), \mathfrak{x}, \mathfrak{t}) & \geq \mathcal{F}(f(\mathfrak{x}), \mathfrak{x}, \mathfrak{t}) \circledast \mathcal{F}\left(\mathfrak{x}_{n}, \mathfrak{x}_{n}, \mathfrak{t}\right) \geq \mathcal{F}\left(f(\mathfrak{x}), \mathfrak{x}_{n}, \mathfrak{t}\right) \circledast \mathcal{F}\left(\mathfrak{x}_{n}, \mathfrak{x}, \mathfrak{t}\right) \\
& \geq \mathcal{F}\left(f(\mathfrak{x}), f\left(\mathfrak{x}_{n-1}\right), \mathfrak{t}\right) \circledast \mathcal{F}(\mathfrak{x}, \mathfrak{x}, \mathfrak{t}) \geq \mathcal{F}\left(\mathfrak{x}, \mathfrak{x}_{n-1}, \mathfrak{t}\right) \circledast \mathcal{F}(\mathfrak{x}, \mathfrak{x}, \mathfrak{t})
\end{aligned}
$$

Therefore, we have $\mathcal{F}(f(\mathfrak{x}), \mathfrak{x}, \mathrm{t})=1$. This means that $f(\mathfrak{x})=\mathfrak{x}$. Hence $\mathfrak{x}$ is a fixed point of $f$.

We now show that $x$ is a unique fixed point $f$. Assume that $x \neq \mathfrak{y}$. Then, we get

$$
\mathcal{F}(\mathfrak{x}, \mathfrak{y}, \mathfrak{t})<\mathcal{F}(f(\mathfrak{x}), f(\mathfrak{y}), \mathfrak{t})=\mathcal{F}(\mathfrak{x}, \mathfrak{y}, \mathfrak{t})
$$

which is a contradiction. Hence, we obtain $\mathfrak{x}=\mathfrak{y}$.

Corollary 1. Let $\left(U, \mathcal{F}_{p}, \circledast\right)$ be a standard PFMS induced by a given complete partial metric $p$ on $U$. If a self-mapping $f$ on $U$ is a partial fuzzy contraction mapping, then $f$ has a unique fixed point in $U$.

Proof. If $\left(U, \mathcal{F}_{p}, \circledast\right)$ is a standard PFMS induced by a given complete partial metric $p$ on $U$, then we know that $\left(U, \mathcal{F}_{p}, \circledast\right)$ is a complete PFMS where $\mathcal{F}_{p}(\mathfrak{x}, \mathfrak{y}, \mathfrak{t})=\frac{\mathfrak{t}}{\mathfrak{t}+p(\mathfrak{x}, \mathfrak{y})}$ for all $\mathfrak{x}, \mathfrak{y} \in U$ and $\mathfrak{t}>0$. Also, we have $\lim _{t \rightarrow \infty} \mathcal{F}_{p}(\mathfrak{x}, \mathfrak{y}, \mathfrak{t})=1$. So, all conditions of Theorem 4 are satisfied and it is obtained that $f$ has a unique fixed point in $U$. 
The next example is given to illustrate the validity of Theorem 4 .

Example 4. Let $U=\mathbb{R}^{+}, \mathfrak{a} \circledast \mathfrak{b}=\mathfrak{a} \mathfrak{b}$ for all $\mathfrak{a}, \mathfrak{b} \in[0,1]$ and the mapping $\mathcal{F}: U \times U \times(0, \infty) \rightarrow[0,1]$ be defined by

$$
\mathcal{F}(\mathfrak{x}, \mathfrak{y}, \mathrm{t})=\frac{\mathrm{t}}{\mathrm{t}+\max \{\mathfrak{x}, \mathfrak{y}\}} .
$$

It is known that $(U, \mathcal{F}, \circledast)$ is a complete PFMS from Example 2 and Proposition 4. Consider the selfmapping $f$ on $U$ defined by $f(x)=\frac{x}{2}$. It is obvious that $f$ is a partial fuzzy contraction mapping. Indeed, we have

$$
\mathcal{F}(f(\mathfrak{x}), f(\mathfrak{y}), k \mathrm{t})=\mathcal{F}(f(\mathfrak{x}), f(\mathfrak{y}), k \mathrm{t})=\mathcal{F}\left(\frac{\mathfrak{x}}{2}, \frac{\mathfrak{y}}{2}, k \mathrm{t}\right)=\frac{k \mathrm{t}}{k \mathrm{t}+\max \left\{\frac{\mathfrak{x}}{2}, \frac{\mathfrak{y}}{2}\right\}}=\frac{k \mathrm{t}}{k \mathrm{t}+\frac{1}{2} \max \{\mathfrak{x}, \mathfrak{y}\}}
$$

for all $\mathfrak{x}, \mathfrak{y} \in \mathbb{R}^{+}$. Therefore, we obtain that $\mathcal{F}(f(\mathfrak{x}), f(\mathfrak{y}), k \mathfrak{t})=\mathcal{F}(\mathfrak{x}, \mathfrak{y}, \mathfrak{t})$ for all $\mathfrak{x}, \mathfrak{y} \in \mathbb{R}^{+}$whenever $k=\frac{1}{2}$. Also, $\lim _{t \rightarrow \infty} \mathcal{F}(\mathfrak{x}, \mathfrak{y}, \mathfrak{t})=\lim _{t \rightarrow \infty} \frac{\mathrm{t}}{\mathrm{t}+\max \{\mathfrak{x}, \mathfrak{y}\}}=1$. Hence all conditions of Theorem 4 are satisfied for $f(x)=\frac{x}{2}$ defined on this space. And one can easily verify that $f$ has a unique fixed point which is $x=0$.

Note that, none of the conditions of Theorem 4 cannot be omitted. The next example shows that the condition to be non-decreasing is not superfluous.

Example 5. Let $U$ be a non-empty set, $a \circledast b=\min \{a, b\}$ and the mapping $\mathcal{F}: U \times U \times(0, \infty) \rightarrow[0,1]$ defined by $\mathcal{F}(\mathfrak{x}, \mathfrak{y}, \mathfrak{t})=e^{-\mathrm{t}}$ for all $\mathfrak{x}, \mathfrak{y}, \mathfrak{z} \in U$ and $\mathrm{t}>0$. It can be shown with a similar proceeding in Example 3 that $(U, \mathcal{F}, \circledast)$ is a PFMS. Furthermore, $(U, \mathcal{F}, \circledast)$ is complete. Also, $\mathcal{F}(f(\mathfrak{x}), f(\mathfrak{y}), k \mathrm{t})=e^{-k \mathrm{t}} \geq e^{-\mathrm{t}}=\mathcal{F}(\mathfrak{x}, \mathfrak{y}, \mathrm{t})$ for all $k \in(0,1), \mathfrak{x}, \mathfrak{y} \in U$ and $\mathrm{t}>0$. Consider the selfmapping $f$ on $U$ given by $f(x)=x$. Clearly, $f$ satisfies the contraction condition of Theorem 4 . But the condition $\lim _{t \rightarrow \infty} \mathcal{F}(\mathfrak{x}, \mathfrak{y}, \mathfrak{t})=1$ for all $\mathfrak{x}, \mathfrak{y} \in U$ does not hold and also every point of $U$ is a fixed point of $f$.

The following example shows that the contraction condition of Theorem 4 is not unnecessary.

Example 6. Let $U=\mathbb{R}^{+}, \mathfrak{a} \circledast \mathfrak{b}=\mathfrak{a} \mathfrak{b}$ for all $\mathfrak{a}, \mathfrak{b} \in[0,1]$ and the mapping $\mathcal{F}: U \times U \times(0, \infty) \rightarrow[0,1]$ defined by

$$
\mathcal{F}(\mathfrak{x}, \mathfrak{y}, \mathfrak{t})=\frac{\mathrm{t}}{\mathfrak{t}+\max \{\mathfrak{x}, \mathfrak{y}\}}
$$

We know that $(U, \mathcal{F}, \circledast)$ is a complete PFMS. Also, the condition $\lim _{t \rightarrow \infty} \mathcal{F}(\mathfrak{x}, \mathfrak{y}, \mathfrak{t})=1$ for all $\mathfrak{x}, \mathfrak{y} \in U$ is hold. Consider the self-mapping $f$ on $U$ given by $f(x)=\mathfrak{x}+1$. The mapping $f$ does not satisfy the contraction condition of Theorem 4 . Also, $f$ has not any fixed point.

Theorem 5. Let $(U, \mathcal{F}, \circledast)$ be a PFMS such that $\mathfrak{b} \geq \mathfrak{c}$ whenever $\mathfrak{a} \circledast \mathfrak{b} \geq \mathfrak{a} \circledast \mathfrak{c}$ for all $\mathfrak{a}, \mathfrak{b}, \mathfrak{c} \in[0,1]$. If a self-mapping $f$ on $U$ is a partial fuzzy Edelstein contractive mapping and there is a point $\mathfrak{x}_{0} \in U$ whose sequence of iterates $\left(f^{n}\left(\mathfrak{x}_{0}\right)\right)$ contains subsequence $\left(f^{n_{i}}\left(\mathfrak{x}_{0}\right)\right)$ such that $\lim _{i \rightarrow \infty} \mathcal{F}\left(f^{n_{i}}\left(\mathfrak{x}_{0}\right), \mathfrak{x}, \mathfrak{t}\right)=$ $\lim _{i \rightarrow \infty} \mathcal{F}\left(f^{n_{i}}\left(\mathfrak{x}_{0}\right), f^{n_{i}}\left(\mathfrak{x}_{0}\right), \mathfrak{t}\right)=\mathcal{F}(\mathfrak{x}, \mathfrak{x}, \mathfrak{t})$, then $\mathfrak{x}=\lim _{i \rightarrow \infty} f^{n_{i}}\left(\mathfrak{x}_{0}\right) \in U$ is a unique fixed point of $f$. 
Proof. Let $(U, \mathcal{F}, \circledast)$ be a PFMS such that $\mathfrak{b} \geq \mathfrak{c}$ whenever $\mathfrak{a} \circledast \mathfrak{b} \geq \mathfrak{a} \circledast \mathfrak{c}$ for all $\mathfrak{a}, \mathfrak{b}, \mathfrak{c} \in[0,1]$. Then $\mathcal{F}(\mathfrak{x}, \mathfrak{y}, \cdot):(0, \infty) \rightarrow[0,1]$ is a non-decreasing function for all $\mathfrak{x}, \mathfrak{y} \in U$. So, we have $\left(U, \mathcal{M}_{\mathcal{F}}, \circledast\right)$ is a fuzzy metric space which is given in Theorem 3. Hence, from the Theorem 2, we obtain that $f$ has a unique fixed point.

Theorem 6. Let $(U, \mathcal{F}, \circledast)$ be a complete PFMS. If a self-mapping $f$ on $U$ is a partial fuzzy $\psi$-contractive mapping, then $f$ has a unique fixed point in $U$.

Proof. Let $\mathfrak{x}_{0} \in U$ and $\mathfrak{x}_{n}=f^{n}\left(\mathfrak{x}_{0}\right)$ for all $n \in \mathbb{N}$. Then for all $t>0$, we have

$$
\mathcal{F}\left(\mathfrak{x}_{n+1}, \mathfrak{x}_{n+2}, \mathfrak{t}\right)=\mathcal{F}\left(f\left(\mathfrak{x}_{n}\right), f\left(\mathfrak{x}_{n+1}\right), \mathfrak{t}\right) \geq \psi\left(\mathcal{F}\left(\mathfrak{X}_{n}, \mathfrak{X}_{n+1}, \mathfrak{t}\right)\right)>\mathcal{F}\left(\mathfrak{x}_{n}, \mathfrak{x}_{n+1}, \mathfrak{t}\right)>\cdots>\mathcal{F}\left(\mathfrak{x}_{0}, \mathfrak{x}_{1}, \mathfrak{t}\right) .
$$

This means that the sequence $\left(\mathcal{F}\left(x_{n}, x_{n+1}, t\right)\right)_{n \in \mathbb{N}}$ is non-decreasing on $(0,1]$. Thus, for a given $\mathrm{t}>0$, there exists a point $\mathfrak{a} \in(0,1]$ such that $\lim _{n \rightarrow \infty} \mathcal{F}\left(\mathfrak{x}_{n}, \mathfrak{x}_{n+1}, \mathfrak{t}\right)=\mathfrak{a}$. From contraction condition,

$$
\mathcal{F}\left(\mathfrak{x}_{n}, \mathfrak{x}_{n+1}, \mathfrak{t}\right) \geq \psi\left(\mathcal{F}\left(\mathfrak{x}_{n-1}, \mathfrak{x}_{n}, \mathrm{t}\right)\right)
$$

Therefore, we have $\psi(\mathfrak{a}) \leq \mathfrak{a}$ However, $\mathfrak{s}<\psi(\mathfrak{s})$ for all $\mathfrak{s} \in(0,1)$ and $\psi$ continuous. Therefore, we must have $\mathfrak{a}=1$. By the triangular inequality we obtain $\lim _{n, m \rightarrow \infty} F\left(\mathfrak{x}_{n}, \mathfrak{x}_{m}, t\right)=1$. Hence $\left(\mathfrak{x}_{n}\right)$ is a Cauchy sequence in $(U, \mathcal{F}, \circledast)$. Since $(U, \mathcal{F}, \circledast)$ is complete the sequence $\left(x_{n}\right)$ is convergent. Thus $\lim _{n \rightarrow \infty} \mathcal{F}\left(\mathfrak{x}_{n}, \mathfrak{x}, \mathrm{t}\right)=\mathcal{F}(\mathfrak{x}, \mathfrak{x}, \mathrm{t})=1=\lim _{n, m \rightarrow \infty} \mathcal{F}\left(\mathfrak{x}_{n}, \mathfrak{x}_{m}, \mathrm{t}\right)$.

$\mathcal{F}(f(\mathfrak{x}), \mathfrak{x}, \mathrm{t}) \geq \mathcal{F}(f(\mathfrak{x}), \mathfrak{x}, \mathrm{t}) \circledast \mathcal{F}\left(\mathfrak{x}_{n+1}, \mathfrak{x}_{n+1}, \mathfrak{t}\right) \geq \mathcal{F}\left(f(\mathfrak{x}), \mathfrak{x}_{n+1}, \mathfrak{t}\right) \circledast \mathcal{F}\left(\mathfrak{x}_{n+1}, \mathfrak{x}, \mathfrak{t}\right)$

$=\mathcal{F}\left(f(\mathfrak{x}), f\left(\mathfrak{x}_{n}\right), \mathrm{t}\right) \circledast \mathcal{F}\left(\mathfrak{x}_{n+1}, \mathfrak{x}, \mathrm{t}\right) \geq \psi\left(\mathcal{F}\left(\mathfrak{x}, \mathfrak{x}_{n}, \mathrm{t}\right)\right) \circledast \mathcal{F}\left(\mathfrak{x}_{n}, \mathfrak{x}, \mathrm{t}\right) \geq \mathcal{F}\left(\mathfrak{x}, \mathfrak{x}_{n}, \mathrm{t}\right) \circledast \mathcal{F}\left(\mathfrak{x}_{n}, \mathfrak{x}, \mathrm{t}\right)$

If we take limit both sides as $n \rightarrow \infty$, we have

$$
\lim _{n \rightarrow \infty} \mathcal{F}(f(\mathfrak{x}), \mathfrak{x}, \mathfrak{t}) \geq \lim _{n \rightarrow \infty} \mathcal{F}\left(\mathfrak{x}, \mathfrak{x}_{n}, \mathfrak{t}\right) \circledast \lim _{n \rightarrow \infty} \mathcal{F}\left(\mathfrak{x}, \mathfrak{x}_{n}, \mathfrak{t}\right)=1 \circledast 1=1
$$

Thus $f(\mathfrak{x})=\mathfrak{x}$. Assume that $\mathfrak{x} \neq \mathfrak{y} \in U$ such that $f(\mathfrak{y})=\mathfrak{y}$. Then we have

$$
\mathcal{F}(\mathfrak{x}, \mathfrak{y}, \mathfrak{t})=\mathcal{F}(f(\mathfrak{x}), f(\mathfrak{y}), \mathfrak{t}) \geq \psi(\mathcal{F}(\mathfrak{x}, \mathfrak{y}, \mathfrak{t}))>\mathcal{F}(\mathfrak{x}, \mathfrak{y}, \mathfrak{t}) .
$$

which is a contradiction. Thus, $\mathfrak{x}$ is a unique fixed point of $f$.

Corollary 2. Let $(U, \mathcal{F}, \circledast)$ be a PFMS and the mapping $\psi_{k}:[0,1] \rightarrow[0,1]$ be defined by

$$
\psi_{k}(t)=\frac{t}{t+k(1-t)}
$$

where $k \in(0,1)$. Then, $\psi_{k} \in \Psi$. In this case, every partial fuzzy $\psi_{k}$-contractive mapping is a partial fuzzy contractive mapping.

Proof. If $f$ is a partial fuzzy $\psi_{k}$-contractive mapping on $U$, then $\mathcal{F}(f(\mathfrak{x}), f(\mathfrak{y}), \mathfrak{t}) \geq \psi_{k}(\mathcal{F}(\mathfrak{x}, \mathfrak{y}, \mathfrak{t}))$ for all $\mathfrak{x}, \mathfrak{y} \in U$ and $\mathrm{t}>0$. From this inequality, we obtain that $\mathcal{F}(f(\mathfrak{x}), f(\mathfrak{y}), \mathfrak{t}) \geq \frac{\mathcal{F}(\mathfrak{x}, \mathfrak{y}, \mathrm{t})}{\mathcal{F}(x, \mathfrak{y}, \mathfrak{t})+k(1-\mathcal{F}(\mathfrak{x}, \mathfrak{y}, \mathrm{t}))}$ for all $\mathfrak{x}, \mathfrak{y} \in U$ and $\mathrm{t}>0$. Hence, it is easily seen that $k\left(\frac{1}{\mathcal{F}(x, \mathfrak{y}, \mathfrak{t})}-1\right) \geq \frac{1}{\mathcal{F}(f(\mathfrak{x}), f(\mathfrak{y}), \mathfrak{t})}-1$ for all $\mathfrak{x}, \mathfrak{y} \in U$ and $\mathrm{t}>0$. This shows that $f$ is a partial fuzzy contractive mapping on $U$.

Corollary 3. Let $\left(U, \mathcal{F}_{p}, \circledast\right)$ be the standard PFMS induced by a given partial metric $p$ on $U$ Then the self-mapping $f$ on $U$ is a partial contractive mapping if and only if $f$ is a partial fuzzy contractive mapping. 
Proof. If we assume that $f$ is a partial contractive mapping on $U$, then there exists a $k \in(0,1)$ such that $p(f(\mathfrak{x}), f(\mathfrak{y})) \leq k p(\mathfrak{x}, \mathfrak{y})$ for all $\mathfrak{x}, \mathfrak{y} \in U$. Hence, we have that

$$
k\left(\frac{1}{\mathcal{F}_{p}(\mathfrak{x}, \mathfrak{y}, \mathfrak{t})}-1\right)=k\left(\frac{t+p(\mathfrak{x}, \mathfrak{y})}{t}-1\right)=\frac{k p(\mathfrak{x}, \mathfrak{y})}{t} \geq \frac{p(f(\mathfrak{x}), f(\mathfrak{y}))}{t}=\frac{1}{\mathcal{F}(f(\mathfrak{x}), f(\mathfrak{y}), \mathfrak{t})}-1
$$

for all $\mathfrak{x}, \mathfrak{y} \in U$ and $\mathfrak{t}>0$. This shows that $f$ is a partial fuzzy contractive mapping. The vice versa is obvious with similarly.

\section{CONCLUSION}

In several branches of mathematics and other disciplines such as optimizations, computer science, economics, engineering and etc., the notion of (partial) metric spaces and its generalizations to the fuzzy environment with fixed point theory has a wide range of applications. Because of the significance of these concepts, we aim to study some fundamental fixed point theorems in partial fuzzy metric spaces in the sense of Sedghi et al. Defining some contractive/contraction mappings similar to the Banach contraction mappings in the fuzzy sense, we show that these mappings have a unique fixed point under some conditions. We also give some examples to illustrate the validity of these theorems. The obtained fixed point theorems lead to further investigations and applications. For example, it is possible to extend our results to the case of coupled fixed points in partial fuzzy metric spaces or the other senses of partial fuzzy metric spaces.

\section{ACKNOWLEDGEMENTS}

We would like to thank the referees for their valuable comments.

\section{REFERENCES}

Altun I, Sola F, Simsek H, 2010. Generalized contractions on partial metric spaces. Topology and its Applications, 157(18):2778-2785.

Başarır M, Şahin A, 2017. Some results of the new iterative scheme in hyperbolic space. Communications of the Korean Mathematical Society, 32(4):1009-1024.

Bukatin M, Kopperman R, Matthews S, Pajoohesh H, 2009. Partial Metric Space. The American Mathematical Monthly, 116(8):708-718.

Czerwik S, 1993. Contraction mappings in b-metric spaces. Acta Mathematica et Informatica Universitatis Ostraviensis, 1(1):5-11.

Gäher S, 1963. 2-metriche raume and ihre topologische structur. Mathematische Nachrichten, 26:115-148.

George A, Veeramani P, 1994. On Some Results in Fuzzy Metric Space. Fuzzy Sets and Systems, 90:365-368

Gregori V, Miñana JJ, Miravet D, 2019. Fuzzy partial metric spaces. International Journal of General Systems, 48(3):260-279.

Gregori V, Morillas S, Sapena A, 2010. On a class of completable fuzzy metric spaces. Fuzzy Sets and Systems 161(16):2193-2205.

Gregori V, Sapena A, 2002. On fixed point theorems in fuzzy metric spaces. Fuzzy Sets and Systems, 125:245252.

Grabiec M, 1988. Fixed point in fuzzy metric space. Fuzzy Sets and Systems, 27(3):385-389.

Haghi RH, Rezapour S, Shahzad N, 2013. Be careful on partial metric fixed point results. Topology and its Applications, 160(3): 450-454.

Kramosil I, Michalek J, 1975. Fuzzy metrics and statistical metric space. Kybernetica, 11(5):336-344.

Kaleva O, Seikkala S, 1984. On fuzzy metric space. Fuzzy Sets and Systems, 12(3):215-229.

Lahiri BK, Das P, Dey LK, 2011. Cantor's theorem in 2-metric spaces and its applications to fixed point problems.

Taiwanese Journal of Mathematics, 15(1):337-352. 
Matthews S, 1994. Partial Metric Topology. Annals of the New York Academy of Sciences-Paper Edition, 728:183-197.

Mihet D, 2004. A Banach contraction theorem in fuzzy metric spaces. Fuzzy Sets and Systems, 144(3):431-439.

Mihet D, 2008. Fuzzy $\psi$-contractive mappings in non-Archimedean fuzzy metric spaces. Fuzzy Sets and Systems, 159(6):739-744.

Mustafa Z, Sims B, 2006. A new approach to generalized metric spaces. Journal of Nonlinear and Convex Analysis, 7(2): 289-297.

O'Neill SJ, 1996. Partial metrics, valuations and domain theory. Annals of the New York Academy of Sciences, 806(1):304-315.

Piera AS, 2001. A contribution to the study of fuzzy metric spaces. Applied General Topology, 2(1):63-75.

Rodriguez-Lopez J, Romaguera S, 2004. The Hausdorff fuzzy metric on compact sets. Fuzzy Sets and Systems, 147(2):273-283.

Sedghi S, Shobkolaei N, Altun I, 2015. Partial fuzzy metric space and some fixed point results. Communications in Mathematics, 23(2):131-142.

Şahin A, Başarır M, Khan SH, 2015. On the g-best proximity point results for G-generalized proximal contraction mappings in G-metric spaces. AIP Coference Proocedings, 1676, 020025.

Valero O, 2005. On Banach fixed point theorems for partial metric spaces. Applied General Topology, 6(2):229240.

Vasuki R, Veeramani P, 2003. Fixed Point Theorems and Cauchy Sequences in Fuzzy Metric Spaces. Fuzzy Sets and Systems, 135(3):415-417.

Yue Y, Gu M, 2014. Fuzzy partial (pseudo-)metric space. Journal of Intelligent and Fuzzy Systems, 27(3):11531159.

Zadeh, LA, 1965. Fuzzy sets. Information and Control, 8(3):338-353. 